\title{
\%. VISIÓN \\ -

\section{Certificado eu organic bio logo para la comercialización internacional de la cebolla junca pulverizada en el corregimiento de Berlín Santander}

$\mathrm{Eu}$ organic bio logo certificate for the international marketing of pulverized junc onion in the small town of Berlin Santander

\author{
Angélica María Carvajal-Guerrero ${ }^{\mathrm{a}^{*}}$, Johanna Milena Mogrovejo-Andrade ${ }^{\mathrm{b}}$, Kesia Daniela Osorio-Piña ${ }^{\mathrm{c}}$ \\ $a^{*}{ }^{M}$ Magister en Finanzas, angelicamariacg@ufps.edu.co, ORCID 0000-0001-7093-0842, Universidad Francisco de Paula Santander, Cúcuta, Colombia. \\ ${ }^{b}$ Doctora en Estudios Politicos, johannamogrovejo@ufps.edu.co, ORCID 0000-0002-9694-3382, Universidad Francisco de Paula Santander, Cúcuta, Colombia. \\ ${ }^{c}$ Profesional enComercio Internacional, kesiadanielaop@ufps.edu.co, Universidad Francisco de Paula Santander, Cúcuta, Colombia.
}

Cómo citar: Carvajal, A. M., Mogrovejo, J. M. \& Osorio, K. D. (2019). Certificado eu organic bio logo para la comercialización internacional de la cebolla junca pulverizada en el corregimiento de Berlín Santander. Revista Visión Internacional, 1(1), 7-11.

Recibido: Agosto 10, 2018; Aceptado: Noviembre 15, 2018

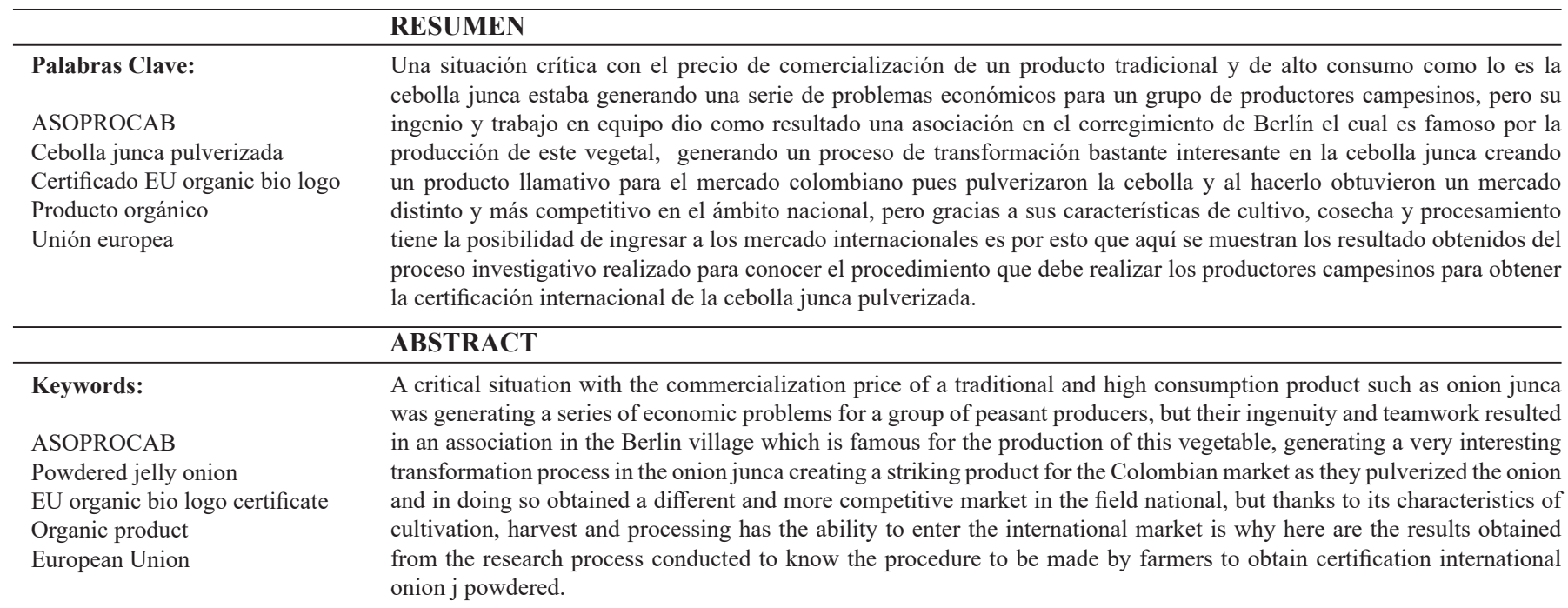

\section{Introducción}

Colombia es un país con una enorme diversidad de climas y pisos térmicos, lo cual le permite cultivar y cosechar una gran variedad de productos hasta tal punto que son representativos en el mercado nacional e internacional, (Trade map.2017).

Entre todos los productos que se cosechan en el país y que presenta grandes posibilidades de obtener el reconocimiento del mercado en el exterior, es la cebolla junca que están procesando los productores campesinos de la Asociación de
Trabajadores, Productores y Comercializadores Agropecuarios de Berlín (ASOPROCAB).

Debido a las diferentes alternativas que ofrece el mercado, la asociación vio la oportunidad de darle un valor agregado a su producción de cebolla junca, es por esto que ellos han generado un proceso de transformación a este vegetal dándole como resultado una nueva presentación de cebolla junca pulverizada, debido a esto los miembros de ASOPROCAB pudieron generar una estabilización de los precios de venta, sumado al hecho de su nueva presentación el vegetal es cultivado de manera orgánica. 
Gracias a las cualidades que presenta la cebolla junca pulverizada, los miembros de la asociación pueden pensar en la posibilidad de vender su producto en los mercados extranjeros, ya que los alimentos orgánicos se han convertido en una tendencia global, agregando a este factor la practicidad del producto para el consumo y la distribución del mismo sin embargo, en este punto ASOPROCAB tiene algunas falencias ya que es necesario certificar su producto como orgánico, para generar veracidad a este hecho.

\section{Materiales y métodos}

De acuerdo a la rigurosa que se debe tener en cuenta en una investigación en donde se aplica el método descriptivo basado en la reseña de las características del objeto a estudiar (Bernal, 2010, p. 60), por lo tanto en el proyecto "Diseño de estrategias para la obtención del certificado EU organic bio logo para la comercialización internacional de la cebolla junca pulverizada en el corregimiento de Berlín Santander" se empleó el método descriptivo, teniendo en cuenta la fundamentación en donde no se deben manipular las variables a estudiar y que por medio de la observación se especifica las características que se tienen en cuenta para en direccionar el buen desarrollo de los objetivos de la investigación (Rosario y Camacho 2015; Osorio 2018).

\section{Requisitos básicos para los procesos de certificación}

Hoy en día debido a los diferentes cambios que se presentan en los mercados nacionales e internacionales y gracias a la globalización, las nuevas tendencias y exigencias de los países es asegurar que los productos que ingresen al territorio sean de buena calidad y estén libres de plagas o cualquier agente que perjudique la salud y bienestar tanto de sus habitantes como de su producción nacional.

Es por esto que las empresas al momento de pensar en comercializar o vender sus productos especialmente aquellos como los del sector agroindustrial deben tener muy claro y en cuenta cuales son las exigencias y certificaciones fitosanitarias y de entrada tiene cada uno de los países que desea incursionar.

En la actualidad la agricultura orgánica presenta dos grandes problemas el primero el primero la certificación o validación, pues dichos procesos son realizados por entes extranjeros y llegan a ser procesos algo costosos; y el segundo la comercialización ya que estos productos aún están siendo vendidos a mercados muy pequeños y donde la competencia internacional es demasiado amplia y los productores mexicanos aún son bastante inexpertos en este mercado donde todos buscan ganar su parte.(Gómez, Gómez y Schwentesius, 2016).

Cuando una empresa u organización desea obtener una certificación ya sea para un producto o servicio enfocado en la manera en la que realiza los procesos en la misma, debe ser cociente que no es una tarea que se pueda realizar a la ligera, ya que cada certificación establece una serie de parámetros, procesos y costos a los cuales la empresa que desee adquirir dicho certificado debe cumplir para poder obtenerlo.

Estos parámetros también permiten a las personas y entes de control saber que si una organización u empresa ha logrado obtener dicho certificado es porque ha llevado a un feliz término un procedimiento que en algunos casos es más extenso y cotoso que en otros. (Osorio, 2018, p. 43).

La certificación y el etiquetado de los productos que pueden ser dependiendo del alcance que se desee (regional, nacional o internacional), todo esto no es únicamente en Latinoamérica, ya que es una tendencia global debido a que el nivel de vida de las personas a aumentado y han generado o añadido una nueva serie de costumbres en diversos aspectos incluyendo la alimentación. (Maldonado, Rivas y Trujillo, 2013).

Según el INVIMA el registro sanitario es un documento de tipo público en el cual la entidad mencionada al principio da la capacidad a la empresa, organización o asociaron de elaborar, comercializar, importar, exportar, envasar, procesar, hidratar y vender una serie de productos con los cuales se debe tener un especial cuidado, (Invima, 2018).

Para esta investigación se establecieron los procedimientos más adecuados que deben cumplir ASOPROCAB para la obtención de los permisos y certificados tanto en el mercado nacional como en el internacional para la comercialización de la cebolla junca pulverizada. Dentro los requisitos o parámetros se encuentran.

El Registro Sanitario, este certificado sirve para demostrar a los consumidores finales (humano, animal) que los productos finales que hayan sido procesados o envasados son aptos para su comercialización y consumo. En el caso de Colombia la entidad encargada de controlar y de generar este documento público es el Instituto Nacional de Vigilancia de Medicamentos y Alimentos (INVIMA).

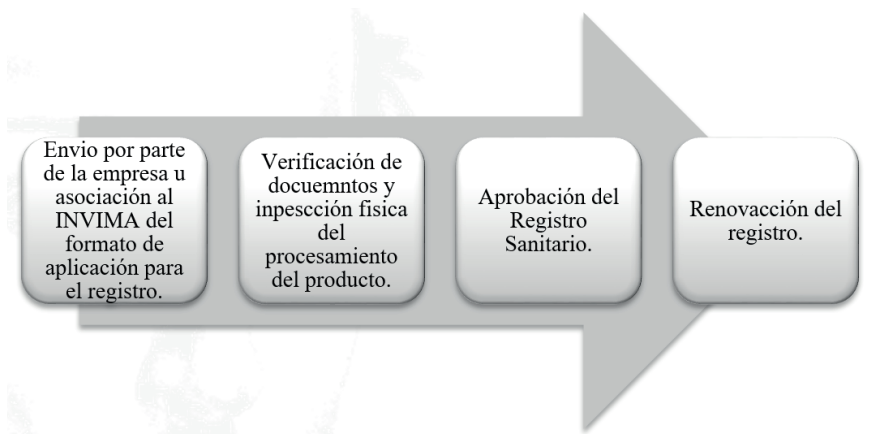

Figura 1. Proceso para obtener el registro sanitario Fuente: Osorio K, 2018, p. 44

Otro de los requisitos o certificaciones se encuentra Las Buenas Prácticas Agrícolas (BPA), se debe tramitar ante el Instituto Colombiano Agropecuario (ICA). Este certificado se le otorga aquellos productores colombianos que cumplan con los parámetros exigidos por la resolución 3002 del 2017, en 
donde se demuestra que el producto final es apto para entregar al consumidor, asegurando su bienestar al momento de consumir las frutas y hortalizas, de igual forma esta resolución indica que las tierras de los agricultores están en condiciones de poder cultivar y cosechar frutas y hortalizas con los más altos estándares de calidad asegurando así el óptimo consumo.

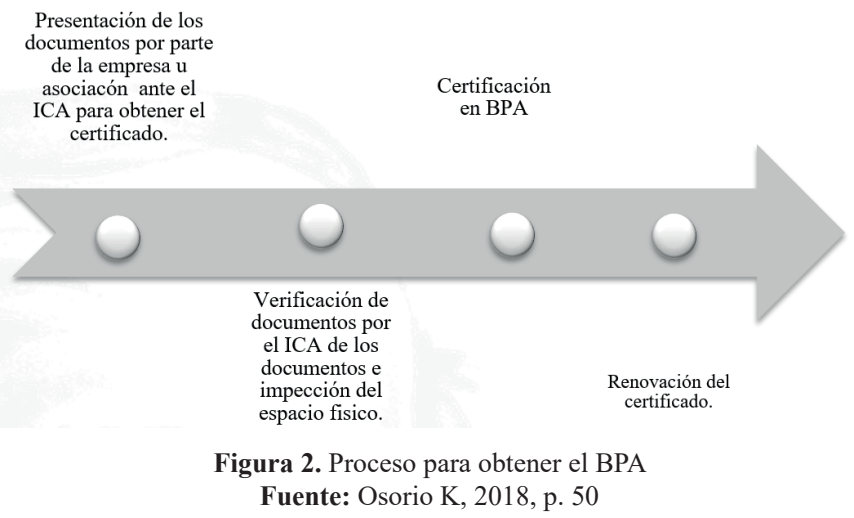

Por último se encuentra el certificado de productor orgánico bajo el certificado EU organic Bio logo, esta certificación la otorga el Ministerio de Agricultura y reglamentada por la resolución 0187 del 2006 (este reglamento establece la producción primaria, procesamiento, empacado, etiquetado y sistemas de control de productos ecológicos) y la resolución 199 del 2016(modifica parcialmente algunos artículos de la resolución 0187 del 2006). (Valencia, 2016).

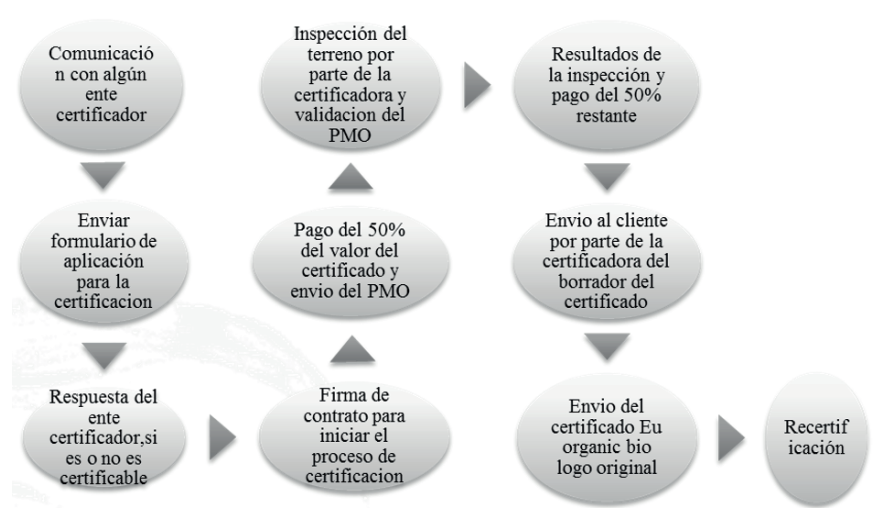

Figura 3. Proceso para obtención del certificado Eu Organic bio logo Fuente: Osorio K, 2018, p. 56

Las empresas que tome la decisión de obtener estos certificados para la comercialización tanto en el mercado nacional como en el mercado internacional, debe estar preparado tanto financieramente como la adecuación que deben realizar para estar de acuerdo a que cada una de las normas especifican; contando también los procesos de capacitación implican un gasto de todos los factores que intervienen en la producción de la cebolla junca sin embargo, al lograr el certificado el producto recibe un aval que para el público consumidor transmite confianza y seguridad (Osorio, 2018, p. 60).

El sector cebollero en Ocaña ha tenido un importante repunte en sus ventas debido a que se ha convertido en un producto típico, el cual es conocido ampliamente en diferentes partes del territorio colombiano; e incluso en algunos países, pero un producto no se vende únicamente por ser un producto reconocido el proceso para lograr que un producto logre ser reconocido a nivel internacional es algo complejo porque lo que en un lugar está bien visto en otro puede ser tomado como una ofensa por ello es clave saber comercializar el producto y poder todas las ventajas de mismo para que sin importar el lugar es logre el mismo impacto.(Delgado, 2016).

\section{Beneficios y costos de la certificación}

(Osorio, 2018) nos indica que dentro de los grandes retos que tiene un emprendedor es poder llegar a ofrecer su producto o servicio a otros países y ver como este va evolucionando con el tiempo logrando así ser más competitivos, para poder llegar a este punto se requiere una serie de esfuerzos no solo de tipo logístico, productivo o económico; ya que al querer presentar y comerciar un producto o servicio en otro país se deben cumplir con las normas establecidas por cada nación y estos parámetros si no son bien conocidos pueden hacer que un excelente producto tenga las puertas totalmente cerradas en un mercado.

Posterior al conocimiento de requerimientos para la obtención de los certificados, se prosiguió con la visita institucional a miembros y directivos de ASOPORCAB, la finalidad de la visita era conocer a través de una entrevista realizada a los directivos de la asociación y una encuesta realizada a 10 miembros de la asociación; conocer cuál es el estado actual de ASOPROCAB y la visión que tiene de su producto y las certificaciones.

Con la entrevista se pudo analizar que el trabajo de cada uno de los miembros ha sido diferente para llegar a lo que son hoy, que la cebolla junca pulverizada ha sido conocida por entes internacionales pero la falta de una capacidad instalada y las debidas certificaciones no han permitido una finalización del proceso de venta al extranjero y que en un futuro próximo darán apertura a su planta de procesamiento lo cual facilitara el proceso de certificación de la cebolla junca pulverizada.

De la encuesta se determinó que los miembros entienden la calidad del producto y comprenden el valor agregado del producto que obtendría el mismo si los certificasen sin embargo, los costos que la asociación debe asumir en total por la obtención de los tres certificados supera los 30 millones de pesos contando los gastos de certificación y los gastos de adecuación a los predios; por ello se concluye que son conscientes de la importancia de las certificaciones, pero necesitan un plan de acción para trabajar sobre él y no presentar a largo plazo problemas de solvencia económica. 
En el énfasis por obtener la certificación se hace necesario profundizar en conceptos que permitirán una mejor comprensión al respecto.

Es el caso de la agricultura orgánica, donde (FAO, 2012) nos dice que es un sistema que se ha creado con la finalidad de reducir o eliminar por completo el uso de productos no renovables en los cultivos con la finalidad de entregar un producto de mayor calidad que proteja el medio ambiente y tenga un impacto positivo mayor en la salud de las personas que consuman los productos obtenidos de estas prácticas.

En cuanto a los alimento orgánico, nos dice Grupo Bimbo (2015) que son aquellos alimentos que se obtiene por medio de técnicas que no afecta de manera agresiva al medio ambiente y de igual manera es sustentada por una legislación o normativa el cual le otorga un logotipo que le permite al consumidor del mismo saber una característica especial del producto.

Cebolla junca pulverizada es vegetal que es ampliamente utilizado en la cocina como condimento y a su vez como una planta medicinal para curar diferentes dolencias, el cual ha sufrido un proceso de transformación en el cual se ha convertido el tallo en polvo. (El tiempo, 2016).

Los certificado fitosanitario por su parte son documentos que se expiden con el único fin de decir que el producto importado o exportado cumple con los requisitos sanitarios estipulados y a su vez está de acuerdo al estipulado por el modelo de certificación CIPF (convección internacional de protección fitosanitaria). (FAO.2012)

La certificación orgánica es aquel conjunto de procesos el cual ratifica que el producto se ha producido bajo estándares de tipo ambiental desde el campo hasta el consumidor, teniendo la capacidad de garantizar todos los procesos que se llevan a cabo. (CERES, 2012)

Certificado organic EU bio logo es el certificado expedido por la unión europea el cual cumple la finalidad de garantizar que cumplas con la normativa que esta misma ha estipulado en el ámbito de producción orgánica. (CERES, 2012)

También el Control de calidad es necesario el cual nos indica que es un conjunto de acciones las cuales garantizan que los procesos realizados con los productos certificados sean idénticos unos de los otros según lo estipulado en la normatividad. (ICA, 2016).

El eco etiqueta es una grafico o símbolo el cual se coloca en el empaque final del producto con la finalidad de mostrar las características medioambientales del mismo hacia el consumidor final. (Biblioteca agrícola estadounidense. 2011)

$\mathrm{Y}$ sin duda alguna a destacar el ICA que es el instituto colombiano agropecuario la cual es una entidad de orden público cuyo objetivo es desarrollar, estipular, y controlar todo lo relacionado con el agro colombiano de igual manera negocia las normas sanitarias y fitosanitarios a nivel bilateral y multilateral, de igual manera el ICA se encarga de garantizar la calidad de todos los productos e insumos que se produzcan en el país. (ICA 2016)

\section{Proponiendo estrategias}

Teniendo toda la información recopilada en la investigación en momentos anteriores, se hicieron una serie de estrategias bajo las cuales el proceso de certificación seria manejable y controlable refiriéndonos no únicamente al aspecto económico sino también logístico y administrativo; entre las estrategias propuestas se expusieron aspectos como, la creación de manuales de cumplimiento para cada uno de los miembros de la asociación, la capacitación tanto de los ítems manejados por cada certificación (registro sanitario, certificado de buenas prácticas agrícolas y certificado Eu organic bio logo) como el cultivo y cosecha de manera orgánica, la creación de un presupuesto basado en los niveles de gastos de adecuamiento promedio y la tarifa del año vigente de la certificación; con esto se busca un proceso más ameno y controlable para los directivos y miembros de ASOPROCAB convirtiendo la certificación de la cebolla junca pulverizada como un producto orgánico internacional sea una realidad posible a largo plazo y no una meta inalcanzable.

\section{Conclusiones}

Alguno de los cambios que en un principio fueron muy bien vistos hoy se están cuestionando seriamente uno de ellos, es la manera en la que los productores campesinos actualmente cosechan las frutas, vegetales y hortalizas que normalmente son encontradas en los supermercados; pues muchas de las semillas, fertilizantes e insecticidas no son de origen natural sino que han sido modificados por la mano del hombre para hacerlos más coloridos, más grandes o hasta más dulces, pero a la larga estos cambios producto no de la adaptación de la planta a un nuevo ambiente sino por parte del hombre ha tenido repercusiones en varias especies de animales y en enfermedades y problemas de salud que las personas no tenían antes de estas modificaciones.

Convertir la información presentada en esta investigación es una tarea que ASOPROCAB debe asumir con total responsabilidad siendo constantes y trabajando de manera organizada como un todo para poder obtener las certificaciones, de igual forma los directivos de la asociación deben buscar entidades de fomento que financien los cotos de certificación o por otro lado entidades financieras que permitan créditos con bajos intereses para que de este modo no presenten problemas de índole económica; la contratación de un ingeniero agrícola (ayude en los proceso de implementación de cada uno de los ítems de las certificaciones y ejerza un control en cada uno de los predios) y un profesional en comercio internacional ( para que apoye en proceso de comercialización internacional de la cebolla junca pulverizada). 


\section{Referencias}

Bernal, C. (2010). Metodología de la investigación. 3rd ed. Bogotá, Colombia: PEARSON EDUCACIÓN, p.60.

CERES Colombia (2017) Certificación Orgánica [blog]. Recuperado de: http://cerescolombia-cert.com/sector-deservicios/certificacion-organica/

Delgado V;(2016). Plan de negocios para la creación de una empresa comercializadora de cebolla cabezona ocañera. (Especialización). Universidad Santo Tomas especialización en gerencia de empresas agropecuaria. Cúcuta. Colombia .Recuperado de: http://repository.usta. edu.co/handle/11634/9567

El tiempo. (2016, 25 febrero). Producen en Santurbán cebolla junca orgánica. EL TIEMPO. Recuperado de http://www. eltiempo.com/archivo/documento/CMS-16520049

FAO (2012). Requisitos para los certificados fitosanitarios. Recuperado de http://www.fao.org/3/Y3241S/y3241s06. htm

Gómez Laura, Gómez Ángel, Schwentesius Rita (2016). Desafíos de la agricultura orgánica comercialización y certificación. Recuperado de: http://ciestaam.edu. $\mathrm{mx} /$ desafios-la-agricultura-organica-certificacioncomercializacion/

Grupo Bimbo. (2016)¿QUÉ ES UN ALIMENTO ORGÁNICO? [Articulo]. Recuperado de: http://www. nutriciongrupobimbo.com/lo-que-debes-saber/paranutrirte/adultos/que-es-un-alimento-organico.html.

Instituto Colombiano Agropecuario. (2017).Wikica glosario agropecuaria [Glosario]. Recuperado de: https:/www. ica.gov.co/El-ICA/Glosario.aspx

INVIMA (2018). Procedimiento de expedición de registros sanitarios, permisos sanitarios, notificaciones sanitarias sin estudio previo y control/revisión posterior. Versión 5. Recuperado de https://www.invima.gov.co/procesos/ archivos/ASS/RSA/ASS-RSA-PR002.pdf

Maldonado, B., Trujillo, M. Y Rivas, L. (2013). Certificación de productos orgánicos en México. (Pregrado). Escuela Superior de Comercio y Administración Unidad Santo Tomás. Instituto Politécnico Nacional México. Recuperado de: http://congreso.investiga.fca.unam.mx/ docs /xviii/docs/13.11.pdf

Osorio, k. (2018). Certificado Eu Organic Bio Logo Para La Comercialización Internacional De La Cebolla Junca Pulverizada En El Corregimiento De Berlín Santander (tesis de pregrado). Universidad Francisco de Paula Santander, Cúcuta, Colombia.

Rosario, M. y Camacho, C. (2015). Apuntes de metodología de la investigación. UNESUR, p.102

Trade Map. (2017) Perspectivas de diversificación de mercado para un producto exportado por Colombia en 2017 Producto: 0703 Cebollas, chalotes, ajos, puerros y demás hortalizas aliáceas. [Estadísticas]. Recuperado de: http://www.trademap.org/Country_SelProductCountry
Graph. $\quad$ aspx?nvpm=3|170||||0703|||4|1|1|2|1|1|2|1|1. spx?nvpm=3|170||||0703|||4|1|1|2|1|1|2|1|1.

Valencia A. (2016). Ministerio de agricultura y desarrollo rural resolución número 00199 de 2016 Recuperado de https://www.minagricultura.gov.co/tramites-servicios/ Documents/Resolucion_199_de_2016.pdf 